\title{
Interval type-2 fuzzy aggregation operator in decision making and its application
}

\author{
M. Lathamaheswari ${ }^{1}$ D. Nagarajan ${ }^{1} \cdot$ J. Kavikumar $^{2} \cdot$ Said Broumi $^{3}$
}

Received: 23 June 2020 / Accepted: 25 January 2021 / Published online: 26 February 2021

(c) The Author(s) 2021

\begin{abstract}
Type-2 fuzzy sets (T2FSs) can deal with higher modeling and uncertainties which exist in the real-world application, specifically in the control systems. Particularly the climate changes are always uncertain and thus, the type- 2 fuzzy controller is an effective system to handle those situations. Polyhouse is a methodology used to cultivate the plants. It breaks the seasonal hurdle of the formulation and it is also suitable for the conflictive climate conditions. Controlling and directing internal parameters of the polyhouse play an essential role in the growth of the plant. Among those, humidity is an important element when one deals with the growth of the plant in polyhouse. It affects the weather, as well as the global change of the climate and hence, the inner climate of the polyhouse will be disturbed. In this paper, operational laws for triangular interval type-2 fuzzy numbers and derived triangular interval type-2 weighted geometric (TIT2WG) operator with their desired mathematical properties using Dombi triangular norms. Also, humidity control is analyzed using interval type-2 fuzzy controller (IT2FC) with the use of derived aggregation operator which is the aim of the paper. Further stability of the system has been analyzed by applying four different defuzzification methods and the method is recommended which gives a better response.
\end{abstract}

Keywords Humidity control $\cdot$ Interval type- 2 fuzzy controller $\cdot$ Polyhouse $\cdot$ Triangular norms

\section{Introduction}

Polyhouse model affected by climate, arrangement of a local building, the required in-house climate and basic components for functioning [10]. Gubbuk and Pekmezci [12] conducted a study to determine the crop and the quality of a special type of banana. It also compared the results of

D. Nagarajan

dnrmsu2002@yahoo.com

M. Lathamaheswari

lathamax@gmail.com

J. Kavikumar

kavi@uthm.edu.my

Said Broumi

broumisaid78@gmail.com

1 Department of Mathematics, Hindustan Institute of Technology and Science, Chennai, India

2 Department of Mathematics and Statistics Faculty of Applied Science and Technology, Universiti Tun Hussein Onn, Parit Raja, Malaysia

3 Laboratory of Information Processing, Faculty of Science Ben M'Sik, University Hassan II, Casablanca, Morocco
$\mathrm{PH}$ and open-field cultivation. Deligeorgidis and Ipsilandis [7] resolved the extent of penetration into the clay for the cucumber and tomato production in polyhouse by Pergande and Thysan.

Lawn management system is generally an informationbased technology and system. It diagnoses, describes and maintains the uncertainties in the field for the highest profitability, and preservability and consequently, it protects the capability of the land. This system anxious with controlling cramped areas within the field instead of doing it in the same field itself and it assumes that the agriculturalist who completely adopts the information can have higher recovery than failing to do so.

Usually, agricultural inputs depend on weather, soil, and also on the demand for the product. Farming in the polyhouse is profitable as compared to the open-field production. Since polyhouse functions are irrespective of the season, and production, it is possible to have quality crop 4-8 times throughout the year than that of the open-field production farming. Therefore higher returns can be achieved in polyhouse. Generally, polyhouse is enclosed by the polythene to gather the light and transfer into the heat [7]. 
In classical polyhouse, the meticulous building controlled the environment by its covering materials namely glass walls and roofs by granting the fast harvesting of solar radiation and converting the agricultural conditions. The components of the polyhouse is useful in maximizing the light transmission of the light and preserving the heat. The humidity, nutrients, and temperature soil should be controlled to reduce the diseases in the crop and at the same time to maximize their quality [13]. Using this way, the horticultural plants can be protected from the unusual weather changes and an internal climate can be standardized [10].

The execution of a normally aired out polyhouse, structured for humidity circumstance, and it had a huge vent ratio of 1.05 [15]. The consequences of cramp approach on carnation based polyhouse in complete randomized design have been studied [8]. Mendel et al. [28], examined the simplicity of interval type-2 fuzzy logic systems. The wheat herb in a basin of soil and aquaculture matured wheat has been compared [9]. Zarandi et al. [54], made a stock market analysis using type-2 fuzzy model. By controlling the elements of the polyhouse, the in-house thermic environment can be regulated [41].

A nursery was of prime importance for the successful house stead program to get the quality crop. Production of an active and healthy plant is the essential thing since it is going to be maintained for a few more months. It also increases the long-term growth in terms of the height of the planet than the outside environment. Nursery planting in the polyhouse enhances the economic condition of the farmer. The advantage of forming polyhouse is that it is suitable for the farmers who are holding small land because the polyhouse can be made in 400-10,000 $\mathrm{m}^{2}$. Those who cannot hold even this much, can form a group among themselves and run the polyhouse. They can get the guidance of the experts on advanced technologies to be used in running the polyhouse [41].

The dissemination of the air and spreading of microclimate by keeping the polyhouse [26]. The economic activity of capsicum and tomato yield with the irrigation system in a normally ventilated polyhouse [42]. A study has been made with two medical yields to calculate the farming performance and compared them according to the lawn condition [38]. A study has been done to compare the morphological growth of different varieties of tomatoes in the open ground using enclosed PH [40]. Cherif [6] examined the performance of type-2 fuzzy logic control for bioinformatics. The polytrenches are highly useful for cultivating the vegetables in the cold desert environments [19]. Biglarbegian et al. [3], examined the stability of type- 1 and interval type- 2 fuzzy logic systems in the area of modeling. The probabilities of cabbage growth under various polyhouse designs and they proposed an optimized structure for the fruitful cabbage production during sub-frost winters in that area [53]. A comparative study of energy flow in the polyhouse and open-field for Iran cucumber manufacture has been done [47]. Castillo and Melin [5] introduced type-2 fuzzy logic systems. Azar [1] have made an overview of type-2 fuzzy logic systems. Mendel and Liu [29] introduced simplified interval type-2 fuzzy logic systems. Zarandi and Gamasaee [55] proposed a type-2 fuzzy system model for reducing bullwhip effects in supply chains and applied in steel manufacturing. Kumbasar [21] analyzed the stability of interval type-2 fuzzy control systems based on PD type. The evolution related to the desirable effects of the structure's administration and its enclosed components on the microclimate [48]. The effects of the province method on the Eggplant crop at the north Khuzestan, Iran [49]. Experiment has been done on thirty ideal farms to evaluate the production cost and the benefits of polyhouse [2]. Risk priority number in model future nodes and analyzed the effects using factor analysis has been evaluated [43]. Computational fluid dynamics method was applied to compare the allowance of the temperature of the inner air and their humidity measure with the assumed [50]. Mauricio et al. [25] done with three type of external perturbation and measures noise using different performance indices.

Because of the closed setting of polyhouse, the humidity was provided regularly by the plant and the soil. When the corresponding humidity of the air in polyhouse was greater than the external climate, the frequency was decreased and the heat change increased. The ventilation decreased the temperature of the air and humidity standards inside the polyhouse. The natural ventilation did not require any energy demand in the application. To stabilize the heat of the polyhouse, an effective ventilation system is needed especially in a hot environment [50].

In recent years polyhouse was developed with advanced technologies to control the system for good quality and quantity yield. Manual or automatic control is followed to regulate the parameters of the environment and they should be maintained in a particular range. The diversity of the electrical support of a material can be measured through the protected humidity sensors. These sensors are connected to the water-circling pump to control the corresponding humidity of the air. The quality of the yield will differ according to the soil and environmental conditions [4]. Roseline [39] examined the unit consumption in fuzzy logic control of washing machine.

Irrigation system was designed according to soil humidity-controlled [4]. Triazophos in polyhouse developed soil on capsicum yield has been examined [14]. The effect of a different locations, and guidance on production to crops in the polyhouse also studied [20], the effect of guaranteed cultivation design of polyhouse and its components [45] studied. Massod [27] designed a smart washing machine using fuzzy logic. Hameed et al. [17], estimated students' academic performance using interval type-2 fuzzy logic systems. Jana et al. [18], made a comparative analysis on 
lead removal using micellar-enhanced ultrafiltration based on type-2 fuzzy logic and response surface methodology. Oscar [36, 37] analyzed improve the control performance in efficiency design to the generalized type 2 fuzzy systems. Cervantes and Castillo [22] done a optimal design of the fuzzy system and statistical comparison using student $t$ test.

Bossalli defended farming unit and employed it in calculating the performance and accuracy for a particular period [30]. In smart polyhouse cultivation IoT setting has been used [51]. Fertility estimation on various Polyhouse in the chilled dusty Ladakh area also has been examined [44]. A review of type- 2 fuzzy control systems and the application of type-2 fuzzy in biomedicine have been done [23, 24]. Edge detection and image extraction using type-2 fuzzy as the advantage of type- 2 fuzzy logic $[31,32]$. The traditional cultivation methods need more time and manpower along with regular monitoring. Whereas, the advanced cultivation methods like polyhouse are effective ways to get increased yields without the interference of uncertain and unpredictable conditions. The fuzzy control system was widely applied in many real-world environments. However, it reveals that the type-2 fuzzy controller (T2FC) was not used earlier. Therefore, it motivated this study to apply T2FC using the derived aggregation operator for humidity control of the polyhouse as it handles uncertain conditions effectively within the specified interval [14]. Wang [52] designed an interval type-2 fuzzy controller. Stammingera et al. [46], done the durability test for washing machines. The stability of the intelligent system using an interval type-2 fuzzy controller can be done effectively [33, 34]. Nagarajan et al. [33, 34] proposed interval type-2 fuzzy logic washing machine. Haghrah [16] proposed a novel Python toolkit fir interval type-2 fuzzy logic systems. Namad and Zare [35] analyzed the stability of interval type-2 control systems in frequency domain. Ontiveros-Robles et al. [11] proposed a fuzzy logic controller according to performance and execution time. Oscar [36, 37] done four type of membership function in the simulation for comparison and analyze to generalized type 2 fuzzy logic system.

From the review, to the best of my knowledge humidity control has not yet been dealt with the use of interval type-2 fuzzy logic controller so far. Type-2 fuzzy logic systems provide opportunity to model levels of uncertainty with the conventional type-1 fuzzy struggles. The extra dimension called footprint of uncertainty gives more degrees of freedom for better representation of uncertainty compared to type-1 fuzzy sets.

The paper is organized as follows. In Sect. 2, some of the basic concepts have been given. In Sect. 3, operational laws for triangular interval type-2 fuzzy numbers and aggregation operator have been derived. In Sect. 4, humidity control in the polyhouse has been analyzed using interval type-2 fuzzy logic controller where triangular interval type-2 fuzzy rules have been and fuzzification has been done using proposed aggregation operator. Also applied four defuzzification methods to analyze the stability of the system. Further drawbacks and advantages of the interval type-2 fuzzy logic controller have been given. In Sect. 5, comparative analysis has been done for the proposed methodology with the existing methods. In Sect. 6, conclusion of the present work is given and the future direction also given.

\section{Basics}

In this section, some of the basic concepts have been given.

\section{Dombi triangular norms}

D o m b i $\quad$ n o r m
$\left(1+\left[\left(\frac{1}{T_{1}}-1\right)^{\delta}+\left(\frac{1}{T_{2}}-1\right)^{\delta}\right]^{\frac{1}{\delta}}\right)^{-1}$. Dombi $\quad$ t-conorm $S\left(T_{1}, T_{2}\right)=T_{1} \oplus T_{2}=$ $\left(1+\left[\left(\frac{1}{T_{1}}-1\right)^{-\delta}+\left(\frac{1}{T_{2}}-1\right)^{-\delta}\right]^{-\frac{1}{\delta}}\right)^{-1}, \delta>1, \forall\left(T_{1}, T_{2}\right)$ $\in[0,1]^{2}[0,1]^{2}$.

Based on the above mentioned triangular norms the following operational laws which may be used as a model of the average behavior of almost any system are established here.

Let $\bar{T}, \bar{T}_{1}, \bar{T}_{2}$ be three TIT2FNs and $\delta>1$ then the following operator can be defined.

\section{Triangular interval type-2 weighted geometric operator}

Let $\bar{T}_{i}=\left(\left[\underline{l_{T_{i}}}, \overline{l_{T_{i}}}\right], m_{T_{i}},\left[\underline{r_{T_{i}}}, \overline{r_{T_{i}}}\right]\right), i=1,2, \ldots, n$ be a set of TIT2FNs and let TIT2WG : $\Delta^{n} \rightarrow \Delta, \quad$ if $\operatorname{TIT} 2 \mathrm{WG}_{\beta}\left(\overline{T_{1}}, \overline{T_{2}}, \ldots, \overline{T_{n}}\right)=\bar{T}_{1}^{\beta_{1}} \otimes{\overline{T_{2}}}^{\beta_{2}} \otimes \cdots . . \otimes{\overline{T_{n}}}^{\beta_{2}}$, then the function TIT2WG is called triangular interval type-2 weighted geometric operator and $\beta$ is the vector of $\bar{T}_{i}=, i=1,2, \ldots, n$ and $\sum_{i=1}^{n} \beta_{i}=1$. As a particular case, if $\beta=(1 / n, 1 / n, \ldots, 1 / n)^{T}$ then TIT2WG operator is abridged to triangular interval type- 2 geometric averaging operator a n d i s d e f i n e d b y $\operatorname{TIT}_{2} \mathrm{WG}_{\beta}\left(\overline{T_{1}}, \overline{T_{2}}, \ldots, \overline{T_{n}}\right)=\left(\overline{T_{1}} \otimes \overline{T_{2}} \otimes \cdots \otimes \overline{T_{n}}\right)^{\frac{1}{n}}$.

\section{Characteristics of the polyhouse}

There are some significant characteristics of polyhouse namely framing weight, enamel, loads of the snow, and fluctuation in temperature. These cause enlargement and deflation of signing and glazing materials. 


\section{Fuzzy logic in polyhouse}

Two types of fuzzy sets, namely type- 1 and type-2. They are widely applied in decision making, Engineering as well as in biological fields namely. T1 handles uncertainties with a crisp output whereas $\mathrm{T} 2$ deals more uncertainties with fuzzy values as the output and produces the crumbly output after defuzzification. Polyhouse methodology is used for the whole year without considering the immediate climate changes. Environmental change is always uncertain regarding its period time. Interval-based impreciseness can be handled effectively using $\mathrm{T} 2$ and therefore it is known as interval type- 2 fuzzy setting.

\section{Components of fuzzy controller (FC)}

Rule base, database and reasoning mechanism are the components of FC and they were used for, selecting the fuzzy rules, defining the membership function and deriving a sensible conclusion based on the rule of fuzzy reasoning.

\section{Components of fuzzy logic system (FLS)}

Rule base, fuzzy inference engine (FIE), fuzzifier and defuzzifier are the four components of fuzzy rules. They were chosen so that the fuzzy logic can showcase human thinking, judgment, and perception. These rules were combined to develop a scaling from crisp inputs to T2FS as outputs. Gaussian fuzzifier was implemented to simplify the computation in the FIE where the MFs in the IF-THEN rules were Gaussian and a mapping from a fuzzy set to the crisp point was calculated for the crisp output.

\section{Operational laws and aggregation operator under triangular interval type-2 fuzzy environment}

In this section, operational laws which may be used as a model of the average behavior of the system and aggregation operator have been derived for triangular interval type-2 fuzzy number using Dombi triangular norms.

\section{Operational laws}

In this section, operational laws are derived for triangular interval type-2 fuzzy numbers using Dombi triangular norms.
Addition

$$
\begin{aligned}
& \bar{T}_{1} \oplus \bar{T}_{2}=\left\{\left[\left(1+\left[\left(\frac{1}{l_{T_{1}}}-1\right)^{-\delta}+\left(\underline{\frac{1}{l_{T_{2}}}-1}\right)^{-\delta}\right]^{-\frac{1}{\delta}}\right)^{-1},\right.\right. \\
& \left.\left(1+\left[\left(\frac{1}{\overline{l_{T_{1}}}}-1\right)^{-\delta}+\left(\frac{1}{\overline{l_{T_{2}}}}-1\right)^{-\delta}\right]^{-\frac{1}{\delta}}\right)^{-1}\right] \\
& \left(1+\left[\left(\frac{1}{m_{T_{1}}}-1\right)^{-\delta}+\left(\frac{1}{m_{T_{2}}}-1\right)^{-\delta}\right]^{-\frac{1}{\delta}}\right)^{-1}, \\
& {\left[\left(1+\left[\left(\frac{1}{r_{T_{1}}}-1\right)^{-\delta}+\left(\underline{\frac{1}{r_{T_{2}}}}-1\right)^{-\delta}\right]^{-\frac{1}{\delta}}\right]^{-1},\right.} \\
& \left.\left.\left\{1+\left[\left(\frac{1}{\overline{r_{T_{1}}}}-1\right)^{-\delta}+\left(\frac{1}{\overline{r_{T_{2}}}}-1\right)^{-\delta}\right]^{-\frac{1}{\delta}}\right)^{-1}\right]\right\}
\end{aligned}
$$

\section{Multiplication}

$$
\begin{gathered}
\bar{T}_{1} \otimes \bar{T}_{2}=\left\{\left[\left(1+\left[\underline{\left.\left.\left(\frac{1}{l_{T_{1}}}-1\right)+\left(\frac{1}{l_{T_{2}}}-1\right)^{\delta}\right]^{\delta}\right]^{\frac{1}{\delta}},}\right.\right.\right.\right. \\
\left.\left(1+\left[\left(\frac{1}{\overline{l_{T_{1}}}}-1\right)^{-1}+\left(\frac{1}{\overline{l_{T_{2}}}}-1\right)^{\delta}\right]^{\frac{1}{\delta}}\right]^{-1}\right]
\end{gathered}
$$

$$
\begin{gathered}
\left(1+\left[\left(\frac{1}{m_{T_{1}}}-1\right)^{\delta}+\left(\frac{1}{m_{T_{2}}}-1\right)^{\delta}\right]^{\frac{1}{\delta}}\right]^{-1}, \\
{\left[\left(1+\left[\left(\frac{1}{r_{T_{1}}}-1\right)^{\delta}+\left(\frac{1}{r_{T_{2}}}-1\right)^{\delta}\right]^{\frac{1}{\delta}}\right)^{-1},\right.} \\
\left.\left.\left(1+\left[\left(\frac{1}{\overline{r_{T_{1}}}}-1\right)^{\delta}+\left(\frac{1}{\overline{r_{T_{2}}}}-1\right)^{\delta}\right]^{\frac{1}{\delta}}\right)^{-1}\right]\right\}
\end{gathered}
$$


Multiplication by an ordinary number

$$
\begin{aligned}
& f . \bar{T}=\left\{\left[\left(1+\left[\left(\frac{1}{l_{T}}-1\right)^{-\delta}\right]^{-f \frac{1}{\delta}}\right]^{-1},\left(1+\left[\left(\frac{1}{\overline{l_{T}}}-1\right)^{-\delta}\right]^{-f \frac{1}{\delta}}\right)^{-1}\right]\right. \\
& \left(1+\left[\left(\frac{1}{m_{T}}-1\right)^{-\delta}\right]^{-f \frac{1}{\delta}}\right)^{-1}, \\
& \left.\left[\left(1+\left[\left(\frac{1}{r_{T}}-1\right)^{-\delta}\right]^{-f \frac{1}{\delta}}\right]^{-1},\left(1+\left[\left(\frac{1}{\overline{r_{T}}}-1\right)^{-\delta}\right]^{-f \frac{1}{\delta}}\right)^{-1}\right]\right\}_{\text {(3) }}
\end{aligned}
$$

\section{Power operation}

$$
\begin{aligned}
& \bar{T}^{f}=\left\{\left[\left(1+\left[\left(\frac{1}{l_{T}}-1\right)^{\delta}\right]^{f \frac{1}{\delta}}\right)^{-1},\left[1+\left[\left(\frac{1}{\overline{l_{T}}}-1\right)^{\delta}\right]^{f \frac{1}{\delta}}\right)^{-1}\right]\right. \\
& \left(1+\left[\left(\frac{1}{m_{T}}-1\right)^{\delta}\right]^{f \frac{1}{\delta}}\right)^{-1}, \\
& \left.\left[\left(1+\left[\left(\frac{1}{r_{T}}-1\right)^{\delta}\right]^{f^{\frac{1}{\delta}}}\right)^{-1},\left(1+\left[\left(\frac{1}{r_{T}}-1\right)^{\delta}\right]^{f \frac{1}{\delta}}\right)^{-1}\right]\right\}
\end{aligned}
$$

\section{Triangular interval type-2 weighted geometric (TIT2WG) operator}

Here TIT2WG operator is derived with its desired mathematical properties which are representing the essential qualities of the optimized control system. With this aggregation operator, the properties namely aggregation of TIT2FNs using TIT2WG operator is again a TIT2FN, Idempotency, associativity, and stability, triangular interval type-2 fuzzy logic carries on with the task of introducing mathematical analysis into logic.

\section{Theorem}

Let $\bar{T}_{i}=\left(\left[l_{T_{i}}, \overline{l_{T_{i}}}\right], m_{T_{i}},\left[r_{T_{i}}, \overline{r_{T_{i}}}\right]\right), i=1,2, \ldots, n$ be a set of TIT2FNs then their aggregated value using TIT2WG operator i s $\quad$ s t i 11 a $\quad$ T I T $2 \mathrm{~F} \mathrm{~N}$, $0 \leq l_{T_{i}} \leq \overline{l_{T_{i}}} \leq m_{T_{i}} \leq r_{T_{i}} \leq \overline{r_{T_{i}}} \leq 1, i=1,2, \ldots, n$ and
$\operatorname{TIT} 2 \mathrm{WG}_{\beta}\left(\overline{T_{1}}, \overline{T_{2}}, \ldots, \overline{T_{n}}\right)$

$=\left\langle\left[\left(1+\operatorname{Add}\left[\left(\frac{1}{l_{T_{i}}}-1\right)^{\delta}\right]^{\beta \frac{1}{\delta}},\left(1+\operatorname{Add}\left[\left(\frac{1}{\bar{l}_{T_{1}}}-1\right)^{-1}\right]^{\beta \frac{1}{\delta}}\right)^{-1}\right]\right.\right.$

$\left(1+\left[\operatorname{Add}\left(\frac{1}{m_{T_{i}}}-1\right)^{\delta}\right]^{\beta \frac{1}{\delta}}\right)^{-1}$,

$\left[\left(1+\left[\operatorname{Add}\left(\frac{1}{r_{T_{i}}}-1\right)^{\delta}\right]^{\beta \frac{1}{\delta}}\right)^{-1}\right.$,
$\left.\left.\left(1+\left[\operatorname{Add}\left(\frac{1}{\overline{r_{T_{i}}}}-1\right)^{\delta}\right]^{\beta \frac{1}{\delta}}\right)^{-1}\right]\right\rangle$,

where $\sum_{i=1}^{n}=$ Add.

Proof To prove the theorem, use mathematical induction method.

For $n=2$, using Eq. (4),

$\bar{T}_{1}^{p}=\left\langle\left[\left(1+\left[\left(\frac{1}{l_{T_{1}}}-1\right)^{\delta}\right]^{p \frac{1}{\delta}}\right]^{-1},\left(1+\left[\left(\frac{1}{\overline{l_{T_{1}}}}-1\right)^{\delta}\right]^{p \frac{1}{\delta}}\right)^{-1}\right]\right.$

$\left(1+\left[\left(\frac{1}{m_{T_{1}}}-1\right)^{\delta}\right]^{p \frac{1}{\delta}}\right)^{-1}$,

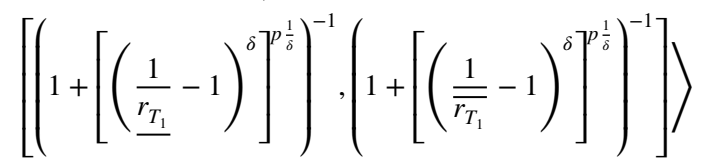

$\bar{T}_{2}^{p}=\left\langle\left[\left(1+\left[\left(\frac{1}{l_{T_{2}}}-1\right)^{\delta}\right]^{p \frac{1}{\delta}}\right)^{-1},\left(1+\left[\left(\frac{1}{\overline{l_{T_{2}}}}-1\right)^{\delta}\right]^{\frac{1}{\delta}}\right)^{-1}\right]\right.$ 


$$
\begin{aligned}
& \left(1+\left[\left(\frac{1}{m_{T_{2}}}-1\right)^{\delta}\right]^{p \frac{1}{\delta}}\right)^{-1}, \\
& \left.\left[\left(1+\left[\left(\frac{1}{r_{T_{2}}}-1\right)^{\delta}\right]^{p \frac{1}{\delta}}\right)^{-1},\left(1+\left[\left(\frac{1}{\overline{r_{T_{2}}}}-1\right)^{\delta}\right]^{p \frac{1}{\delta}}\right)^{-1}\right]\right\rangle
\end{aligned}
$$$$
\operatorname{TIT}_{2} \mathrm{WG}_{\beta}\left(\overline{T_{1}}, \overline{T_{2}}\right)={\overline{T_{1}}}^{\beta_{1}} \otimes{\overline{T_{2}}}^{\beta_{2}}
$$

Let

$$
\begin{aligned}
M_{i} & =\left(\frac{1}{l_{T_{i}}}-1\right)^{\delta}, N_{i}=\left(\frac{1}{\overline{l_{T_{i}}}}-1\right)^{\delta}, O_{i}=\left(\frac{1}{m_{T_{i}}}-1\right)^{\delta}, \\
P_{i} & =\left(\frac{1}{r_{T_{i}}}-1\right)^{\delta}, Q_{i}=\left(\frac{1}{\overline{r_{T_{i}}}}-1\right)^{\delta}
\end{aligned}
$$$$
=\left\langle\left[1+\left[\operatorname{Add}\left(\frac{1}{\left[\left(1+M_{i}\right)^{\frac{\beta_{i}}{\delta}}\right]^{-1}}-1\right)\right]^{\delta}\right]^{\beta_{i} \frac{1}{\delta}},\right.
$$

$$
\left.\left.1+\left[\operatorname{Add}\left(\frac{1}{\left[\left(1+N_{i}\right)^{\frac{\beta_{i}}{\delta}}\right]^{-1}}-1\right)\right]^{\delta}\right]^{\beta_{i} \frac{1}{\delta}}\right]
$$

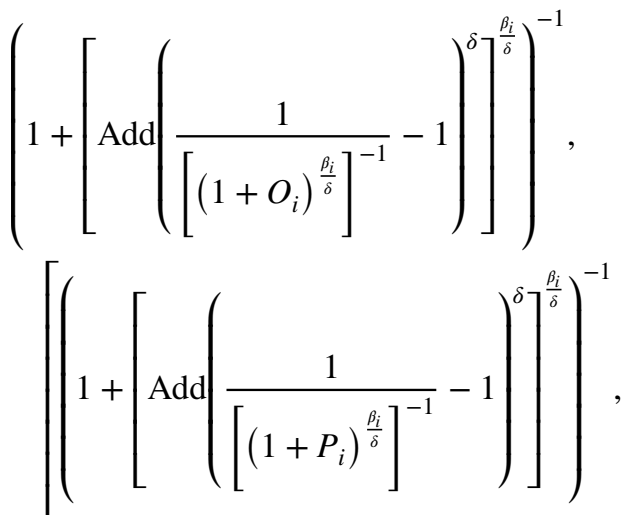

$$
\begin{aligned}
& \left.\left.\left(1+\left[\operatorname{Add}\left(\frac{1}{\left[\left(1+Q_{i}\right)^{\frac{\beta_{i}}{\delta}}\right]^{-1}}-1\right)\right]^{\delta}\right]^{\frac{\beta_{i}}{\delta}}\right]\right\rangle \\
= & \left\{\left[\left(1+\left[\operatorname{Add}\left(M_{i}\right)\right]^{\frac{\beta_{i}}{\delta}}\right)^{-1},\left(1+\left[\operatorname{Add}\left(N_{i}\right)\right]^{\frac{\beta_{i}}{\delta}}\right)^{-1}\right],\right. \\
& \left(1+\left[\operatorname{Add}\left(O_{i}\right)\right]^{\frac{\beta_{i}}{\delta}}\right)^{-1}, \\
& {\left.\left[\left(1+\left[\operatorname{Add}\left(P_{i}\right)\right]^{\frac{\beta_{i}}{\delta}}\right)^{-1},\left(1+\left[\operatorname{Add}\left(Q_{i}\right)\right]^{\frac{\beta_{i}}{\delta}}\right)^{-1}\right]\right\}, }
\end{aligned}
$$

where Add $=\sum_{i=1}^{2}$.

For $n=k$,

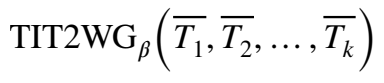

$$
\begin{aligned}
= & \left\langle\left[\left(1+\left[\operatorname{Add}\left(M_{i}\right)\right]^{\frac{\beta_{i}}{\delta}}\right)^{-1},\left(1+\left[\operatorname{Add}\left(N_{i}\right)\right]^{\frac{\beta_{i}}{\delta}}\right)^{-1}\right],\right. \\
& \left(1+\left[\operatorname{Add}\left(O_{i}\right)\right]^{\frac{\beta_{i}}{\delta}}\right)^{-1}, \\
& {\left.\left[\left(1+\left[\operatorname{Add}\left(P_{i}\right)\right]^{\frac{\beta_{i}}{\delta}}\right)^{-1},\left(1+\left[\operatorname{Add}\left(Q_{i}\right)\right]^{\frac{\beta_{i}}{\delta}}\right)^{-1}\right]\right\rangle, }
\end{aligned}
$$

where Add $=\sum_{i=1}^{k}$.

For $n=k+1$

$\operatorname{TIT}_{2} \mathrm{WG}_{\beta}\left(\overline{T_{1}}, \overline{T_{2}}, \ldots, \overline{T_{k+1}}\right)$ 
Complex \& Intelligent Systems (2021) 7:1695-1708

1701

$$
\begin{aligned}
= & \left\langle\left[\left(1+\left[\operatorname{Add}\left(M_{i}\right)\right]^{\frac{\beta_{i}}{\delta}}\right)^{-1},\left(1+\left[\operatorname{Add}\left(N_{i}\right)\right]^{\frac{\beta_{i}}{\delta}}\right)^{-1}\right],\right. \\
& \left(1+\left[\operatorname{Add}\left(O_{i}\right)\right]^{\frac{\beta_{i}}{\delta}}\right)^{-1}, \\
& {\left.\left[\left(1+\left[\operatorname{Add}\left(P_{i}\right)\right]^{\frac{\beta_{i}}{\delta}}\right)^{-1},\left(1+\left[\operatorname{Add}\left(Q_{i}\right)\right]^{\frac{\beta_{i}}{\delta}}\right)^{-1}\right]\right\rangle \otimes } \\
\langle & {\left[\left(1+\left[M_{k+1}\right]^{\frac{\beta_{k+1}}{\delta}}\right)^{-1},\left(1+\left[N_{k+1}\right]^{\frac{\beta_{k+1}}{\delta}}\right)^{-1}\right], } \\
& \left(1+\left[O_{k+1}\right]^{\frac{\beta_{k+1}}{\delta}}\right)^{-1}, \\
& {\left.\left[\left(1+\left[P_{k+1}\right]^{\frac{\beta_{k+1}}{\delta}}\right)^{-1},\left(1+\left[Q_{k+1}\right]^{\frac{\beta_{k+1}}{\delta}}\right)^{-1}\right]\right\rangle, }
\end{aligned}
$$

where the term Add $=\sum_{i=1}^{k}$

$$
\begin{aligned}
= & \left\langle\left[\left(1+\left[\operatorname{Add}\left(M_{i}\right)\right]^{\frac{\beta_{i}}{\delta}}\right)^{-1},\left(1+\left[\operatorname{Add}\left(N_{i}\right)\right]^{\frac{\beta_{i}}{\delta}}\right)^{-1}\right],\right. \\
& \left(1+\left[\operatorname{Add}\left(O_{i}\right)\right]^{\frac{\beta_{i}}{\delta}}\right)^{-1}, \\
& {\left.\left[\left(1+\left[\operatorname{Add}\left(P_{i}\right)\right]^{\frac{\beta_{i}}{\delta}}\right)^{-1},\left(1+\left[\operatorname{Add}\left(Q_{i}\right)\right]^{\frac{\beta_{i}}{\delta}}\right)^{-1}\right]\right\rangle, }
\end{aligned}
$$

where Add $=\sum_{i=1}^{k+1}$.

Hence the result holds for all the values of $n$.

Theorem (idempotency)

Let $\bar{T}_{i}=\left(\left[\underline{l_{T_{i}}}, \overline{l_{T_{i}}}\right], m_{T_{i}},\left[\underline{r_{T_{i}}}, \overline{r_{T_{i}}}\right]\right), i=1,2, \ldots, n$ be a set of TIT2FNs, $0 \leq l_{T_{i}} \leq \overline{l_{T_{i}}} \leq \overline{m_{T_{i}}} \leq r_{T_{i}} \leq \overline{r_{T_{i}}} \leq 1, i=1,2, \ldots, n$. If all $\overline{T_{i}}, i=1,2, \ldots, n$ are equal ie., $\overline{T_{i}}=\bar{T}$ then

$$
\text { TIT } 2 \mathrm{WG}_{\beta}\left(\overline{T_{1}}, \overline{T_{2}}, \ldots, \overline{T_{n}}\right)=\bar{T} \text {. }
$$

Proof Using Theorem 3.2.1,

$\operatorname{TIT}_{2} \mathrm{WG}_{\beta}\left(\overline{T_{1}}, \overline{T_{2}}, \ldots, \overline{T_{n}}\right)$

$$
=\left\langle\left[ 1+\left[\operatorname{Add}\left(\frac{1}{l_{T_{i}}}-1\right)^{\delta}\right]^{\frac{\beta}{\delta}},\left(1+\left[\operatorname{Add}\left(\frac{1}{\overline{l_{T_{1}}}}-1\right)^{-1}\right]^{\delta}\right]\right.\right.
$$

$$
\begin{aligned}
& \left(1+\left[\operatorname{Add}\left(\frac{1}{m_{T_{i}}}-1\right)^{\delta}\right]^{\frac{\beta}{\delta}},\right. \\
& {\left[\left(1+\left[\operatorname{Add}\left(\frac{1}{r_{T_{i}}}-1\right)\right]^{\delta}\right]^{\frac{\beta}{\delta}},\right.} \\
& \left.\left(1+\left[\operatorname{Add}\left(\frac{1}{\overline{r_{T_{i}}}}-1\right)^{\delta}\right]^{\frac{\beta}{\delta}}\right]\right\rangle \\
& =\left\langle\left[\left(1+\left[M_{i}\right]^{\frac{\operatorname{Add}\left(\beta_{i}\right)}{\delta}}\right)^{-1},\left(1+\left[N_{i}\right]^{\frac{\operatorname{Add}\left(\beta_{i}\right)}{\delta}}\right)^{-1}\right],\right. \\
& \left(1+\left[O_{i}\right]^{\frac{\operatorname{Add}\left(\beta_{i}\right)}{\delta}}\right)^{-1} \\
& \left.\left[\left(1+\left[P_{i}\right]^{\frac{\operatorname{Add}\left(\beta_{i}\right)}{\delta}}\right)^{-1},\left(1+\left[Q_{i}\right]^{\frac{\operatorname{Add}\left(\beta_{i}\right)}{\delta}}\right)^{-1}\right]\right\rangle \\
& =\left\langle\left[\left(1+\left[M_{i}\right]^{\frac{1}{\delta}}\right)^{-1},\left(1+\left[N_{i}\right]^{\frac{1}{\delta}}\right)^{-1}\right],\left(1+\left[O_{i}\right]^{\frac{1}{\delta}}\right)^{-1},\right. \\
& \left.\left[\left(1+\left[P_{i}\right]^{\frac{1}{\delta}}\right)^{-1},\left(1+\left[Q_{i}\right]^{\frac{1}{\delta}}\right)^{-1}\right]\right\rangle \\
& =\left\langle\left[\left(1+[M]^{\frac{1}{\delta}}\right)^{-1},\left(1+[N]^{\frac{1}{\delta}}\right)^{-1}\right],\left(1+[O]^{\frac{1}{\delta}}\right)^{-1},\right. \\
& \left.\left[\left(1+[P]^{\frac{1}{\delta}}\right)^{-1},\left(1+[Q]^{\frac{1}{\delta}}\right)^{-1}\right]\right\rangle
\end{aligned}
$$

$=\left\langle\left[\left(1+[M]^{\frac{1}{\delta}}\right)^{-1},\left(1+[N]^{\frac{1}{\delta}}\right)^{-1}\right],\left(1+[O]^{\frac{1}{\delta}}\right)^{-1}\right.$, $\left.\left[\left(1+[P]^{\frac{1}{\delta}}\right)^{-1},\left(1+[Q]^{\frac{1}{\delta}}\right)^{-1}\right]\right\rangle$

$=\left(\left[\underline{l_{T}}, \overline{l_{T}}\right], m_{T},\left[\underline{r_{T}}, \overline{r_{T}}\right]\right)$

$=\bar{T}$,

where $\sum_{i=1}^{n}=$ Add.

Hence the theorem.

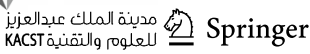




\section{Theorem (associativity)}

Let $\bar{T}_{i}=\left(\left[l_{T_{i}}, \overline{l_{T_{i}}}\right], m_{T_{i}},\left[r_{T_{i}}, \overline{r_{T_{i}}}\right]\right), i=1,2,3$ be three TIT$2 \mathrm{FNs}, \quad 0 \leq l_{T_{i}} \leq \overline{l_{T_{i}}} \leq m_{T_{i}} \leq r_{T_{i}} \leq \overline{r_{T_{i}}} \leq 1, i=1,2, \ldots, n$. The property $\left(\overline{T_{1}} \otimes \overline{T_{2}} \otimes \overline{T_{3}}\right)=\left(\overline{T_{1}} \otimes \overline{T_{2}}\right) \otimes \overline{T_{3}}$ is hold using TIT2WG operator.

Proof Consider the RHS.

$\left(\overline{T_{1}} \otimes \overline{T_{2}}\right) \otimes \overline{T_{3}}$

$\begin{aligned}= & \left\langle\left[\left(1+\left[\operatorname{Add}\left(M_{i}\right)\right]^{\frac{\beta_{i}}{\delta}}\right)^{-1},\left(1+\left[\operatorname{Add}\left(N_{i}\right)\right]^{\frac{\beta_{i}}{\delta}}\right)^{-1}\right],\right. \\ & \left(1+\left[\operatorname{Add}\left(O_{i}\right)\right]^{\frac{\beta_{i}}{\delta}}\right)^{-1}, \\ & {\left.\left[\left(1+\left[\operatorname{Add}\left(P_{i}\right)\right]^{\frac{\beta_{i}}{\delta}}\right)^{-1},\left(1+\left[\operatorname{Add}\left(Q_{i}\right)\right]^{\frac{\beta_{i}}{\delta}}\right)^{-1}\right]\right\rangle } \\ \otimes & \left(\left[l_{T_{3}}, \overline{l_{T_{3}}}\right], m_{T_{3}},\left[\underline{r_{T_{3}}}, \overline{r_{T_{3}}}\right]\right) \\ = & \left\langle\left[\left(1+\left[\operatorname{Add}\left(M_{i}\right)+M_{3}\right]^{\frac{\beta_{i}}{\delta}}\right)^{-1},\left(1+\left[\operatorname{Add}\left(N_{i}\right)+N_{3}\right]^{\frac{\beta_{i}}{\delta}}\right)^{-1}\right],\right. \\ & \left(1+\left[\operatorname{Add}\left(O_{i}\right)+O_{3}\right]^{\frac{\beta_{i}}{\delta}}\right)^{-1}, \\ & {\left.\left[\left(1+\left[\operatorname{Add}\left(P_{i}\right)+P_{3}\right]^{\frac{\beta_{i}}{\delta}}\right)^{-1},\left(1+\left[\operatorname{Add}\left(Q_{i}\right)+Q_{3}\right]^{\frac{\beta_{i}}{\delta}}\right)^{-1}\right]\right\rangle, }\end{aligned}$ where $\sum_{i=1}^{2}=$ Add.

$=\left\langle\left[\left(1+\left[\operatorname{Add}\left(M_{i}\right)\right]^{\frac{\beta_{i}}{\delta}}\right)^{-1},\left(1+\left[\operatorname{Add}\left(N_{i}\right)\right]^{\frac{\beta_{i}}{\delta}}\right)^{-1}\right]\right.$, $\left(1+\left[\operatorname{Add}\left(O_{i}\right)\right]^{\frac{\beta_{i}}{\delta}}\right)^{-1}$,

$\left.\left[\left(1+\left[\operatorname{Add}\left(P_{i}\right)\right]^{\frac{\beta_{i}}{\delta}}\right)^{-1},\left(1+\left[\operatorname{Add}\left(Q_{i}\right)\right]^{\frac{\beta_{i}}{\delta}}\right)^{-1}\right]\right\rangle$,

where $\sum_{i=1}^{3}=$ Add

$=\left(\overline{T_{1}} \otimes \overline{T_{2}} \otimes \overline{T_{3}}\right)$

Hence the theorem.

\section{Theorem (stability)}

Let $\bar{T}_{i}=\left(\left[l_{T_{i}}, \overline{l_{T_{i}}}\right], m_{T_{i}},\left[r_{T_{i}}, \overline{T_{T_{i}}}\right]\right), i=1,2, \ldots, n$ be a collection of TIT 2 FNs. If $v>0$ then

$\mathrm{TIT} 2 \mathrm{WG}_{\beta}\left({\overline{T_{1}}}^{v} \otimes{\overline{T_{n+1}}}_{T_{2}}^{v} \otimes{\overline{T_{n+1}}}, \ldots,{\overline{T_{n}}}^{v} \otimes \overline{T_{n+1}}\right)$

$=\left[\operatorname{TIT} 2 \mathrm{WG}_{\beta}\left(\overline{T_{1}}, \overline{T_{2}}, \ldots, \overline{T_{n}}\right)\right]^{v} \otimes \overline{T_{n+1}}$.

Proof Let the following result to be proved to conclude the result.

To prove

$$
\begin{aligned}
& \quad\left[\operatorname{TIT}_{2} \mathrm{WG}_{\beta}\left(\overline{T_{1}}, \overline{T_{2}}, \ldots, \overline{T_{n}}\right)\right] \otimes \overline{T_{n+1}} \\
& \quad=\operatorname{TIT}_{2} \mathrm{WG}_{\beta}\left(\overline{T_{1}} \otimes \overline{T_{n+1}}, \overline{T_{2}} \otimes \overline{T_{n+1}}, \ldots, \overline{T_{n}} \otimes \overline{T_{n+1}}\right)
\end{aligned}
$$

$\mathrm{LHS}=\left[\operatorname{TIT}_{2} \mathrm{WG}_{\beta}\left(\overline{T_{1}}, \overline{T_{2}}, \ldots, \overline{T_{n}}\right)\right] \otimes \overline{T_{n+1}}$

$$
\begin{aligned}
= & \left\langle\left[\left(1+\left[\operatorname{Add}\left(M_{i}\right)\right]^{\frac{\beta_{i}}{\delta}}\right)^{-1},\left(1+\left[\operatorname{Add}\left(N_{i}\right)\right]^{\frac{\beta_{i}}{\delta}}\right)^{-1}\right],\right. \\
& \left(1+\left[\operatorname{Add}\left(O_{i}\right)\right]^{\frac{\beta_{i}}{\delta}}\right)^{-1}, \\
& {\left.\left[\left(1+\left[\operatorname{Add}\left(P_{i}\right)\right]^{\frac{\beta_{i}}{\delta}}\right)^{-1},\left(1+\left[\operatorname{Add}\left(Q_{i}\right)\right]^{\frac{\beta_{i}}{\delta}}\right)^{-1}\right]\right\rangle }
\end{aligned}
$$

$\otimes\left\langle\left[\left(1+\left[M_{n+1}\right]^{\frac{\beta_{n+1}}{\delta}}\right)^{-1},\left(1+\left[N_{n+1}\right]^{\frac{\beta_{n+1}}{\delta}}\right)^{-1}\right]\right.$, $\left(1+\left[O_{n+1}\right]^{\frac{\beta_{n+1}}{\delta}}\right)^{-1}$,

$$
\left.\left[\left(1+\left[P_{n+1}\right]^{\frac{\beta_{n+1}}{\delta}}\right)^{-1},\left(1+\left[Q_{n+1}\right]^{\frac{\beta_{n+1}}{\delta}}\right)^{-1}\right]\right\rangle,
$$

where $\sum_{i=1}^{3}=$ Add

$=\left\langle\left[\left(1+\left[\operatorname{Add}\left(M_{i}\right)+M_{n+1}\right]^{\frac{\beta_{i}}{\delta}}\right)^{-1},\left(1+\left[\operatorname{Add}\left(N_{i}\right)+N_{n+1}\right]^{\frac{\beta_{i}}{\delta}}\right)^{-1}\right]\right.$, $\left(1+\left[\operatorname{Add}\left(O_{i}\right)+O_{n+1}\right]^{\frac{\beta_{i}}{\delta}}\right)^{-1}$,

$\left.\left[\left(1+\left[\operatorname{Add}\left(P_{i}\right)+P_{n+1 i}\right]^{\frac{\beta_{i}}{\delta}}\right)^{-1},\left(1+\left[\operatorname{Add}\left(Q_{i}\right)+Q_{n+1}\right]^{\frac{\beta_{i}}{\delta}}\right)^{-1}\right]\right\rangle$

$\mathrm{RHS}=\mathrm{TIT} 2 \mathrm{WG}_{\beta}\left(\overline{T_{1}} \otimes \overline{T_{n+1}}, \overline{T_{2}} \otimes \overline{T_{n+1}}, \ldots, \overline{T_{n}} \otimes \overline{T_{n+1}}\right)$. Let 
Fig. 1 The polyhouse control system
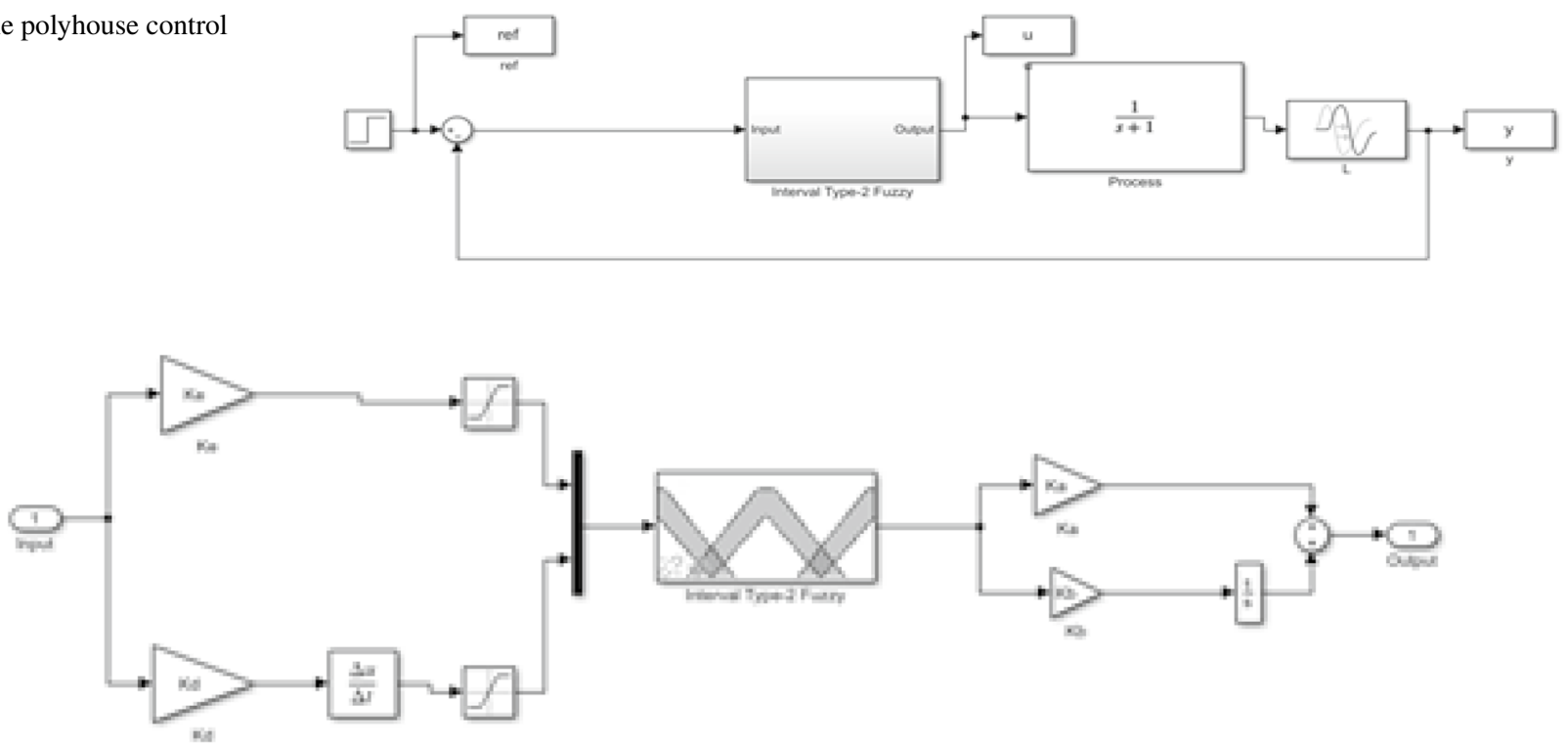

Fig. 2 The T2FS input system

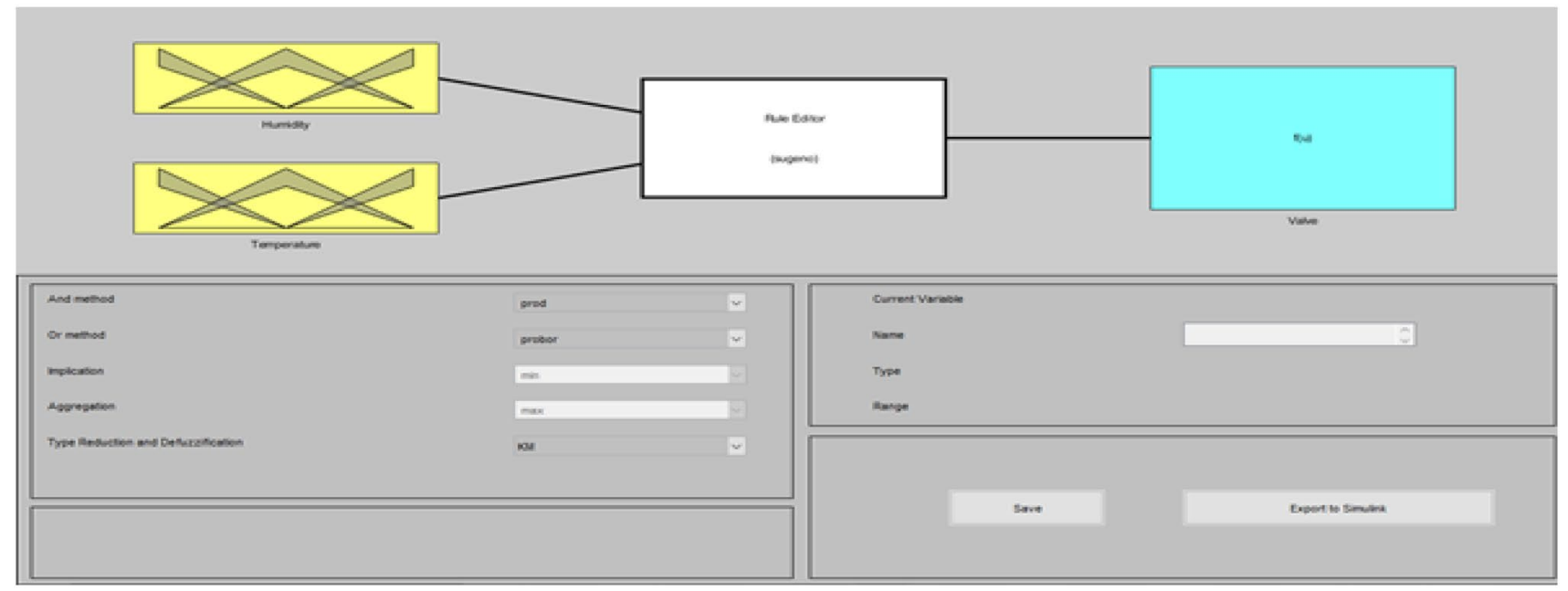

Fig. 3 Interval T2FC inference system

$$
\begin{aligned}
& {\left[\left(1+\sum_{j=i, n+1} M_{j}\right)^{\frac{\beta_{j}}{\delta}}\right]=M_{j}^{*},\left[\left(1+\sum_{j=i, n+1} N_{j}\right)^{\frac{\beta_{j}}{\delta}}\right]} \\
& =N_{j}^{*},\left[\left(1+\sum_{j=i, n+1} O_{j}\right)^{\frac{\beta_{j}}{\delta}}\right]=O_{j}^{*},\left[\left(1+\sum_{j=i, n+1} P_{j}\right)^{\frac{\beta_{j}}{\delta}}\right]=P_{j}^{*} \\
& {\left[\left(1+\sum_{j=i, n+1} R_{j}\right)^{\frac{\beta_{j}}{\delta}}\right]=R_{j}^{*}}
\end{aligned}
$$

Table 1 Rule of the inference system

\begin{tabular}{lll}
\hline Humidity & Temperature & Valve control \\
\hline$L^{\bullet}$ & $L^{\bullet}$ & $L^{\bullet}$ \\
$L^{\bullet}$ & $M^{\bullet}$ & $M^{\bullet}$ \\
$L^{\bullet}$ & $H^{\bullet}$ & $M^{\bullet}$ \\
$M^{\bullet}$ & $L^{\bullet}$ & $M^{\bullet}$ \\
$M^{\bullet}$ & $M^{\bullet}$ & $H^{\bullet}$ \\
$M^{\bullet}$ & $H^{\bullet}$ & $M^{\bullet}$ \\
$H^{\bullet}$ & $L^{\bullet}$ & $M^{\bullet}$ \\
$H^{\bullet}$ & $M^{\bullet}$ & $H^{\bullet}$ \\
$H^{\bullet}$ & $H^{\bullet}$ & $H^{\bullet}$ \\
\hline
\end{tabular}

Where $L^{\bullet}=$ low, $M^{\bullet}=$ Medium, $H^{\bullet}=$ high 
$=\left\langle\left[1+\left[\operatorname{Add}\left(\frac{1}{M_{j}^{*-1}}-1\right)^{\delta}\right]^{\frac{\beta_{i}}{\delta}}\right)^{-1},\left(1+\left[\operatorname{Add}\left(\frac{1}{N_{j}^{*-1}}-1\right)^{\delta}\right]^{\frac{\beta_{i}}{\delta}}\right)^{-1}\right],\left[1+\left[\operatorname{Add}\left(\frac{1}{M_{j}^{*-1}}-1\right)^{\delta}\right]^{\frac{\beta_{i}}{\delta}}\right)^{-1}$,

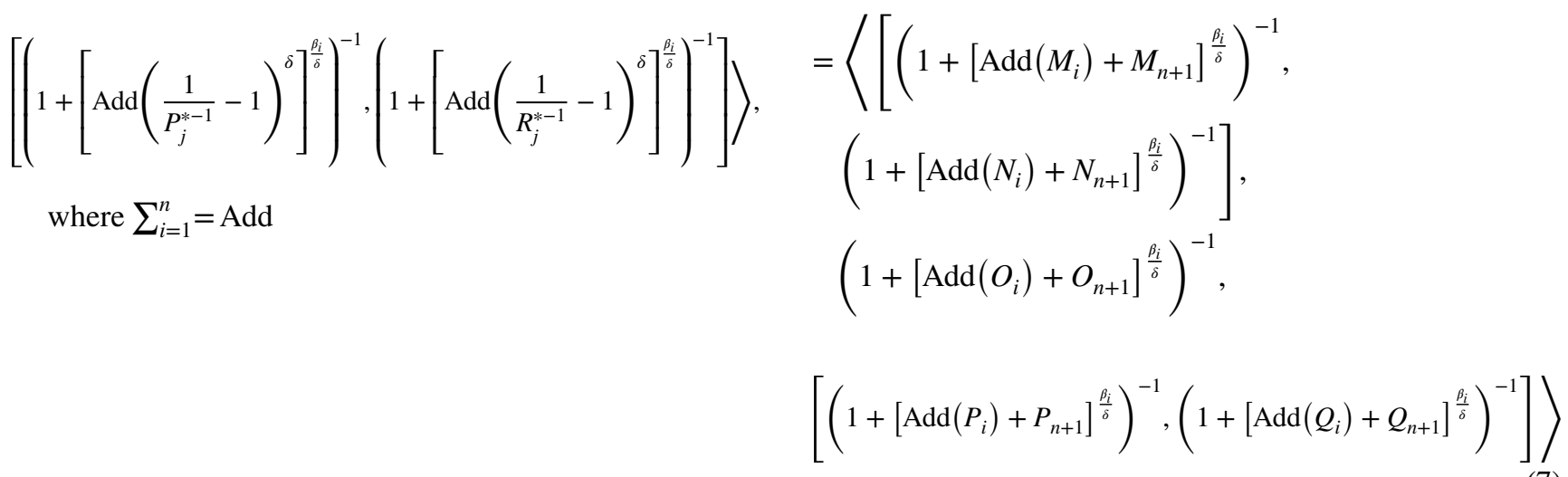

Fig. 4 Stabilized system with response

Fig. 5 Precision
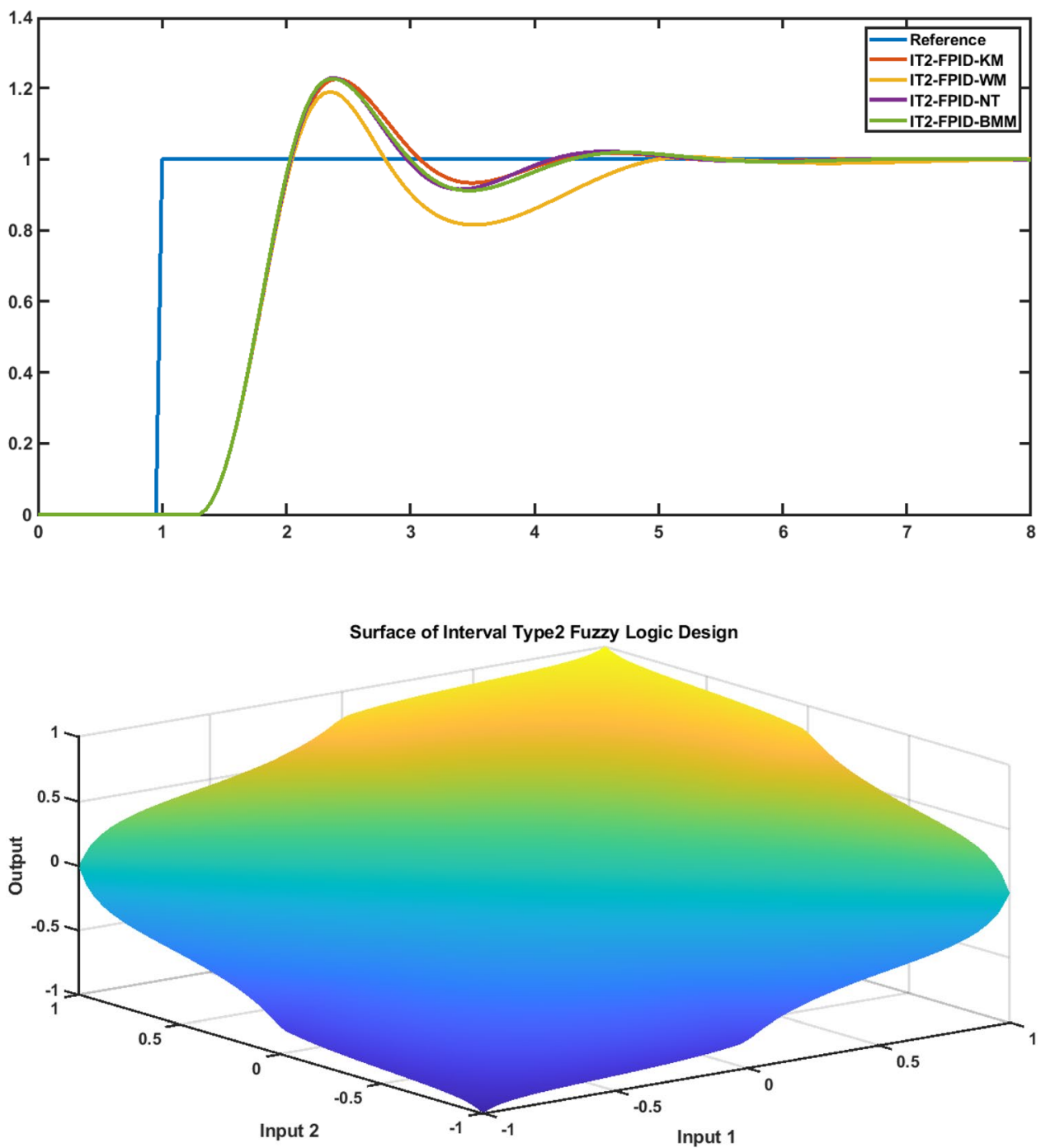
Table 2 Comparative analysis

\begin{tabular}{ll}
\hline Existing methods & Advantage \\
\hline Roselin [39] & Which is capable of having automatic inputs and desired output using type-1 fuzzy logic controller \\
Massod [27] & Analysis has been done for fixing washing time according to the type of cloths \\
Stammingera et al. [46] & $\begin{array}{l}\text { The parameters are crisp numbers and the desired output is obtained in terms of percentage and } \\
\text { the standard units like temperature }\end{array}$ \\
Interval type-2 fuzzy logic controller has been used to automate the amount of detergent intake \\
and stability has been analyzed using four different defuzzification methods and concluded the \\
better method. Also the parameters have been considered as triangular interval type-2 fuzzy \\
numbers and the desired output is obtained using the score values of the inputs \\
Humidity control in the polyhouse has been analyzed \\
Proposed method & Using interval type-2 fuzzy logic control system \\
& Based on the proposed aggregation operator
\end{tabular}

From Eqs. (6) and (7), the result Eq. (5) is true.

Using weighted geometric rule, the following result can be considered and to be proved.

To prove

$\operatorname{TIT} 2 \mathrm{WG}_{\beta}\left({\overline{T_{1}}}^{v},{\overline{T_{2}}}^{v}, \ldots,{\overline{T_{n}}}^{v}\right)=\left[\operatorname{TIT}^{2} \mathrm{WG}_{\beta}\left(\overline{T_{1}}, \overline{T_{2}}, \ldots, \overline{T_{n}}\right)\right]^{v}$

Let

$M_{i}=\left(\frac{1}{l_{T_{i}}}-1\right)^{\delta}, N_{i}=\left(\frac{1}{\overline{l_{T_{i}}}}-1\right)^{\delta}, O_{i}=\left(\frac{1}{m_{T_{i}}}-1\right)^{\delta}$,

$P_{i}=\left(\frac{1}{r_{T_{i}}}-1\right)^{\delta}, Q_{i}=\left(\frac{1}{\overline{r_{T_{i}}}}-1\right)^{\delta}$.

L e t $\quad\left[\left(1+M_{i}\right)^{v \delta}\right]=M_{i}^{*}, \quad\left[\left(1+N_{i}\right)^{v \delta}\right]=N_{i}^{*}$, $\left[\left(1+O_{i}\right)^{v \delta}\right]=O_{i}^{*},\left[\left(1+P_{i}\right)^{v \delta}\right]=P_{i}^{*},\left[\left(1+Q_{i}\right)^{v \delta}\right]=Q_{i}^{*}$.

Consider the LHS,

$$
\begin{aligned}
& \quad \operatorname{TIT} \mathrm{WG}_{\beta}\left({\overline{T_{1}}}^{v},{\overline{T_{2}}}^{v}, \ldots,{\overline{T_{n}}}^{v}\right) \\
& =\left\langle\left[\left(1+\left[\operatorname{Add}^{\prime}\left(1+M_{i}^{*}\right)\right]^{\frac{\beta_{i}}{\delta}}\right)^{-1},\left(1+\left[\operatorname{Add}^{\prime}\left(1+N_{i}\right)\right]^{\frac{\beta_{i}}{\delta}}\right)^{-1}\right]\right. \\
& \left.\left[\left(1+\left[\operatorname{Add}^{\prime}\left(1+P_{i}^{*}\right)\right]^{\frac{\beta_{i}}{\delta}}\right)^{-1},\left(1+\left[\operatorname{Add}^{\prime}\left(1+Q_{i}^{*}\right)\right]^{\frac{\beta_{i}}{\delta}}\right)^{-1}\right]\right\rangle \\
& =\left\langle\left[\left(1+\left[\operatorname{Add}^{\prime}\left(M_{i}\right)\right]^{\frac{\nu \beta_{i}}{\delta}}\right)^{-1},\left(1+\left[\operatorname{Add}^{\prime}\left(N_{i}\right)\right]^{\frac{\nu \beta_{i}}{\delta}}\right)^{-1}\right],\right. \\
& \\
& \left(1+\left[\operatorname{Add}^{\prime}\left(Q_{i}\right)\right]^{\frac{\nu \beta_{i}}{\delta}}\right)^{-1},
\end{aligned}
$$

where $\sum_{i=1}^{u}=$ Add'. $^{\prime}$

$$
\mathrm{RHS}=\left[\operatorname{TIT}^{i=1} \mathrm{WG}_{\beta}\left(\overline{T_{1}}, \overline{T_{2}}, \ldots, \overline{T_{n}}\right)\right]^{v}
$$

$$
\begin{aligned}
& =\left\langle\left[\left(1+\left[\operatorname{Add}\left(M_{i}\right)\right]^{\frac{\beta_{i}}{\delta}}\right)^{-v},\left(1+\left[\operatorname{Add}\left(N_{i}\right)\right]^{\frac{\beta_{i}}{\delta}}\right)^{-v}\right]\right. \\
& =\left\langle\left[\left(1+\left[\operatorname{Add}^{\prime}\left(M_{i}\right)\right]^{\frac{v \beta_{i}}{\delta}}\right)^{-1},\left(1+\left[\operatorname{Add}^{\prime}\left(N_{i}\right)\right]^{\frac{v \beta_{i}}{\delta}}\right)^{-1}\right],\right.
\end{aligned}
$$

$$
\begin{aligned}
& \left(1+\left[\operatorname{Add}^{\prime}\left(O_{i}\right)\right]^{\frac{v \beta_{i}}{\delta}}\right)^{-1}, \\
& \left.\left[\left(1+\left[\operatorname{Add}^{\prime}\left(P_{i}\right)\right]^{\frac{v \beta_{i}}{\delta}}\right)^{-1},\left(1+\left[\operatorname{Add}^{\prime}\left(Q_{i}\right)\right]^{\frac{v \beta_{i}}{\delta}}\right)^{-1}\right]\right\rangle
\end{aligned}
$$

From Eqs. (9) and (10), the result Eq. (8) is true.

From the results Eqs. (5) and (8), the theorem is true.

\section{Humidity control in polyhouse using interval type-2 fuzzy logic controller}

Type-2 fuzzy sets (T2FSs) offer a higher modeling and better understanding of uncertainties using control systems. Here we used fuzzy rule-based inference system using interval type- 2 triangular fuzzy sets and triangular interval type- 2 weighted aggregation operator.

The block diagram of the polyhouse control system using T2FS is displayed in Fig. 1. It has input, interval type 2 fuzzy block, subsystem block, process plant block $(1 / S+1)$, time interval block $L$ and output $y$.

Figure 2 shows the input and output of the subsystem with type-2 fuzzy logic controller associated PID controller has variables $\mathrm{Ka}, \mathrm{Kb}, \mathrm{Ke}, \mathrm{Kd}$ and integrator. These variables were defined in the program script and it can be modified according to the optimization.

This block gets input from the summing point as error values and delivers the calculated output to the process 
plant. Further, its input error values are nothing but part of the system output value ' $y$ ' subtracted from reference input.

Figure 3 shows that fuzzified parameters were measured through rules and portray the applied Fuzzy inference arrangement within the input system.

Two types of control parameter inputs gave nine potential considerations. The overall valve open rules of the input and output parameters valve are tabulated in Table 1.

\section{Stabilized output with the reference}

The output of the system was stabilized using the Karnik-Mendal algorithm (KM), Nie-tan method (NT), Wu-Mendal uncertainty bound method (WM), and Begain-Melak-Mendalmethod (BMM) through the TR method. The stabilized values of $\mathrm{Ke}=1, \mathrm{Kd}=0.5141$, $\mathrm{Ka}=0.077, \mathrm{~Kb}=7.336$, sampling time $=0.05, K=1 \mathrm{~T}=1.1$ and $L=0.3$

Figure 4 shows that the different types of responses such as Karnik-Mendal algorithm (KM), Nie-tan method (NT), Wu-Mendal uncertainty bound method (WM) and Begain-Melak-Mendal method (BMM) through TR method, respectively versus time with a reference value. Settling time is 4.1 , the rising time is 1.7 , maximum overshoot is 1.2 of $\mathrm{Wu}-$ Mendal uncertainty bound method (WM) has less maximum overshoot and very poor settling time among the other methods. Begain-Melak-Mendal method (BMM) has better than the $\mathrm{Wu}-$ Mendal uncertainty bound method (WM) and more settling time than the Karnik-Mendal algorithm. Nie-tan method (NT) is rising time is good as the tough Karnik-Mendal algorithm rise time having the same maximum overshoot of Karnik-Mendal algorithm but having little bit underdambing Karnik-Mendal algorithm and more settling time to Karnik-Mendal algorithm So among this over dambing, underdambing, rise time and settling time in Karnik-Mendal algorithm gives a better response.

\section{Precision}

Fuzzy rule-based subsets were modified and it reshaped the sub-degree within the subset. Fuzzy knowledge-based logic was included for a much better outcome. The output of the model reveals that when the valve was slightly opened, the sub-level was varied uniformly. In Fig. 5 shows that input 1 is humidity and input 2 is temperature is the range of the current variable is $0-100$, display range is $0-100$. Surface graph reveal that the humidity and temperature is low the valve control is low, humidity is low and temperature is medium, the humidity is low and the temperature is high and also humidity is medium and temperature is low then the valve is partially opened. Humidity is medium and temperature is medium, humidity is high and temperature is medium and also humidity and temperature is high then the output valve is fully opened. Humidity is medium and temperature is high, humidity is high temperature is low then the output valve is partially opened.

\section{Drawbacks of interval type-2 fuzzy controller}

Secondary membership degree of interval type-2 fuzzy model is one which made the related operations and computations are simpler than the conventional methods. Also more accuracy can be obtained. But there is a restriction on the number of input variables and cannot deal with indeterminacy of the data.

\section{Advantage of the present method}

In this paper, aggregation operator is used to aggregate any number of triangular interval type-2 fuzzy input variables to provide a single triangular interval type- 2 fuzzy value. And for defuzzification process four different methods have been used and recommended a better method.

\section{Comparative analysis}

Here, comparative analysis has been done for the proposed methodology with the existing methods to prove the advantage and effectiveness of the interval type- 2 fuzzy logic controller with the support of proposed aggregation operator.

In the area of control system, conventional PID controller has been widely used but it has less capability of dealing with the relation between the input and output parameters, whereas type-2 fuzzy logic controller has a very good capability of analyzing the stability of the system with uncertain parameters. From Table 2, It is found that, stability of the interval type- 2 fuzzy control system has been improved than the type-1 fuzzy control system. These results overlap the proposed result. Hence the proposed methodology can be applied to analyze the stability of the control system suitably in comparison with the existing methods.

\section{Conclusion}

Humidity is one of the important core components of the polyhouse ( $\mathrm{PH}$ ) and it must be controlled carefully to obtain good and quality yields. Conversely, it will spoil the fruitful production. There are many methodologies that have been used to control the humidity of the polyhouse. In this present study, operational laws for triangular interval type-2 fuzzy 
numbers have been derived using Dombi triangular norms. Aggregation operator namely triangular interval type-2 weighted geometric operator using Dombi triangular norms has been derived with its desired mathematical properties in detail. Also, interval type-2 fuzzy controller is used to control the humidity value of the air inside the polyhouse and improved efficiency of the overall system and also humidity control is productively done. Four different defuzzifcation methods have been applied for stability analysis and it is found that Karnik-Mendal algorithm gives a better response of the polyhouse intelligent system. In future, humidity control shall be analyzed using neutrosophic control system.

Acknowledgements This research was supported by the Ministry of Higher Education Malaysia, Malaysia, under FRGS Grant no.: K179.

\section{Compliance with ethical standards}

Conflict of interest The authors declare that they have no conflict of interest.

Ethical approval The article does not contain any studies with human participants or animal performed by any of the authors.

Informed consent Informed consent was obtained from all individual participants included in the study.

Open Access This article is licensed under a Creative Commons Attribution 4.0 International License, which permits use, sharing, adaptation, distribution and reproduction in any medium or format, as long as you give appropriate credit to the original author(s) and the source, provide a link to the Creative Commons licence, and indicate if changes were made. The images or other third party material in this article are included in the article's Creative Commons licence, unless indicated otherwise in a credit line to the material. If material is not included in the article's Creative Commons licence and your intended use is not permitted by statutory regulation or exceeds the permitted use, you will need to obtain permission directly from the copyright holder. To view a copy of this licence, visit http://creativecommons.org/licenses/by/4.0/.

\section{References}

1. Azar AT (2012) Overview of type-2 fuzzy logic systems. Int J Fuzzy SystAppl 2(4):1-28

2. Ban SG, Zanic K, Dumicic G, Raspudic E, Selak GV, Ban D (2014) Growth and yield of grafted cucumbers in soil infested with root-knot nematodes. Chil J Agric Res 74(1):29-34

3. Biglarbegian M, Melek WW, Mendel JM (2011) On the robustness of type-1 and interval type- 2 fuzzy logic systems in modeling. InfSci 181(7):1325-1347

4. Bhosale S, Sonavane SS (2016) Automated monitoring and controlling of polyhouse environment. Int J Adv Res ComputEngTechnol 5(8):2333-2338

5. Castillo O, Melin P (2012) Type-2 fuzzy logic systems. In: recent advances in interval type- 2 fuzzy systems spring briefs in applied sciences and technology 1 . $10.1 \mathrm{c} 007 / 978-3-642-28956-9$
6. Cherif AH (2010) Type-2 fuzzy control for bioinformatics-a systems approach. Int J ComputSciNetwSecur 10(7):69-75

7. Deligeorgidis PN, Ipsilandis CG (2004) Determination of soil depth inhabited by Frankliniella occidentalis (Pergande) and Thrips tabaci Lindeman (Thysan, Thripidae) under greenhouse cultivation. J ApplEntomol 128(2):108-111

8. Dalal SR, Nandre DR, Bharad SG, Swarupa U, Shinde RD (2006) Effect of pinching oncarnation cv. YELLOW SOLAR under polyhouse condition. Int J AgricSci 2(2):356-357

9. Du Toit AGA, Labuschagne MT (2007) A comparison between hydroponics systems and pots for growing wheat in the greenhouse. S Afr J Plant Soil 24(2):120-123

10. Elsner BV, Briassouls D, Waaijenberg D, Mistriotis A, Zabeltitz C, Gratraud J, Russo G, Suay-Cortes R (2000) Review of structural and functional characteristics of greenhouses in European union countries: part 1 design requirements. J Agric Res 75(1):1-16

11. Emanuel O-R, Melin P, Castillo O (2018) Comparative analysis of noise robustness of type 2 fuzzy logic controllers. Kybernetika 54(1):175. https://doi.org/10.14736/Kyb-2018-1-0175

12. Gubbuk H, Pekmezci M (2004) Comparison of open-field and protected cultivation of banana (Musa spp .AAA) in the coastal area of Turkey. N Zeal J Crop HorticSci 32:375-378

13. Girija S, Jethava K, Shamante A (2005) Greenhouse cultivation hot arid area. In: Ideas/RePEc, pp 1-11

14. Guru PN, Patil CS (2018) Dissipation studies of triazophos in/ on polyhouse grown capsicum cropped soil. J EntomolZool Stud 6(1):12-16

15. Harmanto A, Tantau HJ, Salokhe VM (2006) Optimization of ventilation opening area of a naturally ventilated net greenhouse in a human tropical environment. ActaHortic 719:165-172

16. Haghrah AA (2019) PyIT2FLS: a new python toolkit for interval type-2 fuzzy logic systems. arXiv: 1909.10051v1 [eess.SY]

17. Hameed IA, Elhoushy M, Osen OL (2017) Interval type-2 fuzzy logic systems for evaluating students academic performance. In: Computers supported education, pp 420-441

18. Jana DK, Roy K, Dey S (2018) Comparative assessment on lead removal using micellar-enhanced ultrafiltration (MEUF) based on type-2 fuzzy logic and response surface methodology. Sep PurifTechnol 207:28-41

19. Kanwar MS, Saleem MM, Ishfaq AP (2011) Effect of protected structures on cabbage production in freezing winters of Ladakh Region. Indian Hortic J 1(1):23-25

20. Kapuriya VK, Ameta KD, Teli SK, Chittora A, Gathala S, Yadav $S$ (2017) Effect of spacing and training on growth and yield of polyhouse grown cucumber (Cucumis sativus L.). Int J CurrMicrobiolApplSci 6(8):299-304

21. Kumbasar T (2014) Robust Stability analysis of PD type single input interval type-2 fuzzy control systems. In: 2014 IEEE international conference on fuzzy systems, pp 634-639. https://doi. org/10.1109/FUZZ-IEEE.2014.6891616

22. Leticia C, Castillo O (2015) Type-2 fuzzy logic aggregation of multiple fuzzy controllers for airplane flight control. InfSci 354:247-256. https://doi.org/10.1016/j.ins.2015.06.047

23. Lathamaheswari M, Nagarajan D, Kavikumar J, Phang C (2018) A review on type-2 fuzzy controller on control system. J Adv Res Dyn Control Syst 10(11):430-435

24. Lathamaheswari M, Nagarajan D, Udayakumar A, Kavikumar J (2018) Review on type-2 fuzzy in biomedicine. Indian J Public Health Res Dev 9(12):322-326

25. Sanchez MA, Castillo O, Castro JR (2015) Generalized type-2 fuzzy systems for controlling a mobile robot and a performance comparison with interval type- 2 and type-1 fuzzy systems. 
Expert SystAppl 42(14):5904-5914. https://doi.org/10.1016/j. eswa.2015.03.024

26. Majdoubi H, Bouard T, Fatnassi H, Bouirden L (2009) Airflow and microclimate patterns in a one-hectare canary type 1 greenhouse: an experimental and CFD assisted study. Agric For Meteorol 149(6-7):1-32

27. Massod RF (2017) Application of fuzzy logic in design of smart washing machine. In: Systems and control, pp 1-7

28. Mendel JM, John RI, Liu F (2006) Interval type-2 fuzzy logic systems made simple. IEEE Trans Fuzzy Syst 14(6):808-821

29. Mendel JM, Liu X (2013) Simplified interval type-2 fuzzy logic systems. IEEE Trans Fuzzy Syst 21(6):1056-1069

30. Mohamed AAA, El-Nagger MG (2018) Economic analysis of protected cultivation: comparison of vegetables vs. fruit. J AgricSci 10(3):187-196

31. Nagarajan D, Lathamaheswari M, Sujatha R, Kavikumar J (2018) Edge detection on DICOM image using triangular norms in type-2 fuzzy. Int J AdvComputSciAppl 9(11):462-475

32 Nagarajan D, Lathamaheswari M, Kavikumar J, Hamzha A (2018) A type-2 fuzzy in image extraction for DICOM image. Int $\mathrm{J}$ AdvComputSciAppl 9(12):352-362

33. Nagarajan D, Lathamaheswari M, Kavikumar J, Bromui S (2019) Intelligent system stability using type-2 fuzzy controller. Int $\mathbf{J}$ IntegrEng 11(1):270-282

34. Nagarajan D, Lathamaheswari M, Kavikumar J, Deenadayalan E (2019) Interval type-2 fuzzy logic washing machine. Int J Fuzzy Logic IntellSyst 19(4):223-233

35. Namad Z, Zare A (2020) Stability analysis of dynamic interval type-2 control systems in frequency domain. Asian J Control. https://doi.org/10.1002/asjc.2407

36. Oscar C, Cervantes L, Soria J, Sanchez M, Castro JR (2016) A generalized type-2 fuzzy granular approach with applications to aerospace. InfSci 354:165-177. https://doi.org/10.1016/j. ins.2016.03.001

37. Oscar C, Leticia A-A, Castro JR, Garcia-Valdez M (2016) A comparative study of type-1 fuzzy logic systems, interval type- 2 fuzzy logic systems and generalized type-2 fuzzy logic systems in control problems. InfSci 354:257-274. https://doi.org/10.1016/j. ins.2016.03.026

38. Parvej MR, Khan MAH, Awal MA (2010) Phenological development and production potentials of tomato under polyhouse climate. J AgricSci 5(1):19-31

39. Roseline SPL (2016) Unit consumption under fuzzy logic control of washing machine. In: Proceedings of national conference on relevance of research methodology in computer science, Pune, India. ISBN 978-93-84654-10-3

40. Singh B, Sirohi NPS (2006) Protected cultivation of vegetables in India: problems and future prospects. ActaHortic 710:339-342

41. Sengar SH, Kothari S (2008) Economic evaluation of greenhouse for cultivation of rose nursery. Afr J Agric Res 3(6):435-439
42. Sreenivasa MD, Prabhakar BS, Hebber SS, Srinivas V, Prabhakar M (2009) Economic feasibility of vegetable production under polyhouse: a case study of capsicum and tomato. J HorticSci 4(2):148-152

43. Sellapan N, Palanikumar D (2015) Evaluation of risk priority number (RPN) in design failure mode and effects analysis (DFMEA) using factor analysis. Int J ApplEng Res 10(14):34194-34198

44. Singh MC, Kachwaya DS, Kalsi K (2018) Soilless cucumber cultivation under protective structures in relation to irrigation coupled fertigation management, economic viability and potential benefits-a review. Int J CurrMicrobiolApplSci 7(3):2451-2468

45. Santosh DT, Tiwari KN, Singh VK (2017) Influence of different protected cultivation structures on water requirements of winter vegetables. Int J Agric Environ Biotechnol 10(1):93-103

46. Stammingera R, Tecchiob P, Ardenteb F, Mathieuxb F, Niestratha $P$ (2018) Towards a durability test for washing-machines. ResourConservRecycl 131:206-215

47. Teitel M, Montero JI, Baeza E (2012) Greenhouse design: concepts and trends. ActaHortic 952:605-620

48. Tayari E (2013) Effects of tillage methods on eggplant yield in north Khuzeston, Iran. Int J Farm Allied Sci 2(5):102-103

49. Testa R, Trapani AMD, Sgroi F, Tudisca S (2014) Economic sustainability of Italian greenhouse cherry tomato. Sustainability 6:7967-7981

50. Tezcan A, Buyuktas K (2015) Determining of some climate parameters fluid computational dynamic technique in naturally ventilated greenhouse. AkdenizUniversitesiZiraatFakultesiDergisi 28(2):71-76

51. Tundup P, Safal RS, Namgail D, Spaldon S, Hussain A, Namgail D, Kumar Y (2018) Soil fertility assessment under different polyhouse in cold arid Ladakh Region. Int J CurrMicrobiolApplSci $7(2): 2135-2138$

52. Wang X (2018) Interval type-2 fuzzy controller design. Syst Simul Technol 14(4):310-313

53. Yousefi M, Darijani F, Jahangiri AA (2012) Comparing energy flow of greenhouse and open-field cucumber production systems in Iran. Afr J Agric Res 7(4):624-628

54. Zarandi MHF, Neshat E, Turksen IB, Rezaee B (2007) A type-2 fuzzy model for stock market analysis. In: 2007 IEEE international fuzzy systems conference, London, pp 1-6. https://doi. org/10.1109/FUZZY.2007.4295378

55. Zarandi MHF, Gamasaee R (2013) A type-2 fuzzy system model for reducing bullwhip effects in supply chains and its application in steel manufacturing. Sci Iran 20(3):879-899

Publisher's Note Springer Nature remains neutral with regard to jurisdictional claims in published maps and institutional affiliations. 